\title{
RETRACTED ARTICLE: TN-2 Exerts Anti-Inflammatory Effects on LPS-Induced Rat Dorsal Root Ganglion Neurons by Inhibiting TLR4-Mediated NF- $\mathrm{BB}$ and MAPK Pathways
}

\author{
Xiaofeng Teng ${ }^{1} \cdot \mathrm{Na} \mathrm{Wei}^{2} \cdot$ Hong Chen ${ }^{1} \cdot$ Kaihua Zhai $^{2}$
}

Received: 17 June 2015 / Accepted: 14 July 2015 / Published online: 28 July 2015

(C) Springer Science+Business Media New York 2015

This paper has been retracted at the request of the authors and the Editor in Chief because it contains material that has been published before.

$\triangle \quad$ Xiaofeng Teng

tengxiaofengnb@163.com

1 Department of Hand Surgery, Ningbo Sixth Hospital,

Ningbo, Zhejiang 315040, China

2 Department of the Second Neurology, the Third Affiliated Hospital of Xinxiang Medical University, Xinxiang, Henan 453003, China 